\title{
IROS 2022 - Das große Wiedersehen
}

Vom 13. bis 15. Januar 2022 trifft sich die Interventionelle Radiologie im Congress Center in Salzburg zur Dreiländertagung der Deutschen (DeGIR), Österreichischen (ÖGIR) und Schweizerischen (SSVIR) Gesellschaften für interventionelle Radiologie und minimal-invasive Therapie. Nach fast zwei Jahren der Einschränkungen und Online-Meetings wird es endlich zum lang ersehnten Wiedersehen beim Interventionell Radiologischen Olbert-Symposium (IROS) in Österreich kommen. Prof. Dr. Andreas H. Mahnken, Kongresspräsident des IROS 2022 und Vorstandsmitglied der DeGIR, gibt uns in diesem Interview einen Überblick zu allen wissenswerten Punkten beim größten interventionell-radiologischen Kongress im deutschsprachigen Raum.

\section{Unter welchen COVID-19-Bedingungen wird der IROS 2022 in Salzburg stattfin-} den?

Das wichtigste natürlich zuerst - das ganze Organisationsteam freut sich, dass wir uns nach langer Zeit wieder in Präsenz zum wissenschaftlichen Austausch zusammenfinden werden! Angesichts der hohen Impfquote unter klinisch tätigem Personal und der unterstützenden Industrie wird der IROS 2022 unter 2G-Bedingungen geplant.

\section{Interventionelle Radiologie zu Pandemie- zeiten - was hat sich geändert?}

COVID-19-Patienten stellen uns alle vor erhebliche Herausforderungen. Wir sehen bekannte Krankheitsbilder wie Blutungen und Thrombose unter einem neuen klinischen Aspekt. Die Organisation der Betreuung dieser Patienten ist eine erhebliche Herausforderung, nicht nur unter dem Aspekt von Hygienekonzepten und Schutz des Personals. Diesem Themenkomplex wird daher eine eigene Sitzung gewährt, um „Best-Practice“-Beispiele zu geben.

\section{Welche Schwerpunkte erwarten die Kon- gressbesuchenden auf dem IROS 2022?}

Ein wichtiger Schwerpunkt des IROS ist seit jeher die Qualifikation entsprechend der DEGIR- Qualifikation. Hier wird es wieder ein abgestimmtes Weiterbildungsprogramm mit Basis- und Spezialkursen geben, das sich auf die Module $D$ und $F$ in den Basiskursen und die Module $A$ und $C$ in den Spezialkursen fokussiert. Die bekannten Fokuskurse werden sich mit spannenden Themen wie dem Management von vaskulären Malformationen, Schlaganfalltherapie aber auch Mammainterventionen befassen. Insgesamt wird es ein sehr breit angelegtes Themenportfolio in den bewährten Sitzungsformaten geben. Auch die sehr gut angenommenen „Hands-On“-Simulatorkurse werden wieder angeboten werden.

Wird es neue Formate beim IROS 2022 geben?

Als größte Neuerung wird es bei diesem IROS erstmalig am 12.01.2022 einen halben Kongresstag mit einem Repetitorium zur gezielten Vorbereitung auf die deutschsprachige DEGIR- und EBIR-Fachprüfung geben. Hier werden entsprechend des Curriculums gezielt die Prüfungsinhalte und Prüfungsformate vermittelt.

Welche Hot Topics können die Besucherinnen und Besucher erwarten?

Neben der schon angesprochenen Sitzung zum Management von COVID-19-Patienten werden in der „Heiße Eisen“-Sitzung bariatrische Embolisation und die Embolisationstherapie bei Osteoarthritis angegangen. Auch Neuigkeiten für das Management der
Unterschenkel- pAVK werden entsprechend des Weiterbildungsschwerpunktes im DEGIR-Modul A aufgegriffen.

\section{Was wird auf dem IROS 2022 für den Nachwuchs getan beziehungsweise ange- boten?}

Es wird wieder unterschiedliche Angebote für den Nachwuchs geben. Angefangen vom kostenlosen Kongressticket für Studierende über die bewährte Sitzung „Wie schreibe ich ein Paper" steht vor allem das „Flinke-Finger“-Programm im Fokus der Nachwuchsförderung. Darüber hinaus wird es als neues Format eine vom Forum Junge Radiologie organisierte Veranstaltungsgruppe im Rahmen des IROS 2022 geben.

\section{Welche Möglichkeiten der Fort- und Wei- terbildungen wird es für MTRA und Radio- logietechnologen und -technologinnen (kurz: RT) geben?}

In bewährter Weise wird wieder in enger Zusammenarbeit mit den MTRA/RTA-Verbänden aus Deutschland, Österreich und der Schweiz ein eigenes Programm für das technische Personal organisiert. Die Inhalte orientieren sich am Modul 3 der Zertifizierung zur „Fachkraft Interventionelle Radiologie“. Im Rahmen des IROS wird gleichzeitig auch die Möglichkeit zur Prüfung in diesem Modul angeboten. Darüber hinaus sind alle MTRA und RTA zum gesamten Programm herzlich eingeladen. Interventionelle Radiologie ist schließlich ein „Teamsport“, bei dem sich optimale Ergebnisse nur erreichen lassen, wenn ärztliches Personal und technisches Personal sich perfekt ergänzen.

Das Interview führte Univ.-Prof. Dr. Peter Minko aus der DeGIR-Lenkungsgruppe Öffentlichkeitsarbeit. 